\title{
TAUBERIAN THEOREMS AND SLOWLY VARYING FUNCTIONS
}

\author{
BY \\ DAVID DRASIN
}

Introduction. Let $k(x)$ be a fixed $L_{1}(-\infty, \infty)$ function. Then if

$$
\int_{-\infty}^{\infty} k(x-y) f(y) d y
$$

exists (either in the sense of Riemann or Lebesgue) for sufficiently large $x$, we define

$$
g(x)=\int_{-\infty}^{\infty} k(x-y) f(y) d y
$$

All functions discussed are assumed to be real-valued and $g(x)$ will always refer to the function defined by (1).

This paper is concerned with consequences of the Tauberian assumption that

$$
g(x) / f(x) \rightarrow L \quad(x \rightarrow \infty)
$$

where $L>0$. An important role will be played by functions $\psi(x)$ which satisfy

$$
\lim _{x \rightarrow \infty} \psi(x+a) / \psi(x)=1 \text { for every fixed } a
$$

the letter $\psi$ will be reserved exclusively for functions which satisfy (3). In [4] these functions are called slowly varying at $\infty$.

The situation discussed here complements that discussed in several classical papers. For example, J. Karamata [7] showed that if

$$
k(t)=e^{t} e^{-e^{t}}
$$

and if $f$ is positive and increasing, then from

$$
g(x)=e^{\lambda x} \psi(x) \quad(\lambda \geqq 0)
$$

follows

$$
f(x) \sim \frac{1}{\Gamma(\lambda+1)} e^{\lambda x} \psi(x)
$$

where $\psi$ satisfies (3); conversely, $f(x)=[\Gamma(\lambda+1)]^{-1} e^{\lambda x} \psi(x)$ implies that $g(x)$ $\sim e^{\lambda x} \psi(x)$.

Received by the editors July 5, 1967. 
More recently, Feller [3], [4] noted that (4) and (5) are in turn equivalent to

$$
\frac{g(x+a)}{f(x)} \rightarrow \Gamma(\lambda+1) e^{\lambda a}
$$

for every fixed $a$, as $x \rightarrow \infty$. Note that (6) is formally a much stronger condition than (2) (set $a=0$ ); one consequence of our results is that (2) and (6) are in fact equivalent. For purposes of comparison, we record that $\Gamma(\lambda+1)$ is the bilateral Laplace transform of $k$ evaluated at $\lambda$ :

$$
\Gamma(\lambda+1)=\int_{-\infty}^{\infty} e^{-\lambda t} k(t) d t=\mathscr{L} k(\lambda)
$$

The goal of the present paper is to derive (4) and (3) from (2), making minimal assumptions on $k$ and $f$; this is the content of Theorem 1. Examples presented in $\$ 9$ indicate that best results are to be obtained when neither $k$ nor $f$ are allowed to vary sign; thus $k$ and $f$ are assumed nonnegative, and further restrictions will be listed in $\S 1$. A converse to Theorem 1 is the content of $\S 8$.

In $\$ 10$, we use Theorem 1 to derive a recent result due to Edrei and Fuchs [2]. It was this result that provided the impetus for the present work.

The final section is devoted to extending Theorem 1 to the situation that $g(x)$, defined by

$$
g(x)=\int_{0}^{x} k(x-y) f(y) d y
$$

satisfies (2) (because of (1.6), this is not covered by Theorem 1). This is an important special case, since Karamata's own characterization of functions of the form (4) and (3) is that (8) and (2) are satisfied with $f$ nonnegative and continuous, and $k(t)=e^{-s t}(t \geqq 0)$ (the dependence on $\lambda$ and $s$ is given in the statement of Theorem 6 in \$11). Similar characterizations appear in Hardy-Rogosinski [5].

This paper is based on a portion of my doctoral dissertation written at Cornell University; it is a pleasure to acknowledge the generous help and inspiration supplied by Professor Wolfgang Fuchs. I wish also to thank Professors Harry Pollard and Daniel Shea for several helpful suggestions.

\section{Statement of Theorem 1.}

Definition. A measurable function $f(x)$ is in class $\mathscr{M}$ if

(1) $f(x) \geqq 0$;

(2) for every fixed $a$,

$$
f(x)<\mu(a ; f)<\infty \quad(-\infty \leqq x \leqq a)
$$

(3) there exist $\delta>0$ and $x_{0}$ (which depend only on $f$ ) with

$$
f(x)>\delta, \quad x>x_{0} .
$$


Definition. $\mathscr{M}^{\prime}$ consists of those functions $f$ in $\mathscr{M}$ such that there exist $\varepsilon>0$, $a_{0}>0$ and $x_{0}$ (depending only on $f$ ) with

$$
f(t) / f(x)>\varepsilon, \quad x_{0}<x \leqq t \leqq x+a_{0} .
$$

For example, a positive increasing function is in $\mathscr{M}^{\prime}$. The major result is

THEOREM 1. Let $f$ be in $\mathscr{M}^{\prime}$, and assume that $k(t)$ is measurable and satisfies

$$
\mathscr{L} k(s)=\int_{-\infty}^{\infty} e^{-s t} k(t) d t \text { exists if }-\sigma<s<\rho
$$

for some positive $\sigma$ and $\rho$ (it is not excluded that one or perhaps both of $\sigma$ and $\rho$ be infinite) whereas

$$
\begin{aligned}
\mathscr{L} k(\rho) & =\mathscr{L} k(-\sigma)=\infty \\
k(t) & >0 \quad \text { almost everywhere. }
\end{aligned}
$$

Then from

$$
\int_{-\infty}^{\infty} k(x-y) f(y) d y=\{L+o(1)\} f(x) \quad(x \rightarrow \infty)
$$

follows that

$$
f(x)=e^{\lambda x} \psi(x)
$$

where

$$
\text { for every fixed } a, \frac{\psi(x+a)}{\psi(x)} \rightarrow 1 \quad(x \rightarrow \infty) .
$$

In addition, $\lambda(\geqq 0)$ must satisfy

$$
\mathscr{L} k(\lambda)=L
$$

REMARK. If $f$ is assumed to be increasing, it is possible to simplify (1.4) by avoiding any assumptions concerning the bilateral Laplace transform of $k$ for negative values of $s$. Condition (1.5) is used only in the proof of Lemmas 3 and $6 ;(1.6)$ is needed in $\$ \$ 5$ and 6.

With particular choices of the kernel $k$, some of (1.1)-(1.3) may be deleted. For example, the $g(x)$ defined by (1) with any of the kernels mentioned in the introduction always satisfies (1.3), so if $g / f \rightarrow L>0, f$ must also. Conditions (1.1) and (1.2) are needed only to prove Lemma 2 , and often that lemma can be established independently, again making use only of properties of the kernel itself. For example, this can be done directly with any of the particular kernels mentioned thus far, as well as with one of the kernels considered in $\$ 10$. 
Since (1.7) is an asymptotic relation, the inequalities derived will be valid only for large values of $x$; we abbreviate this by "for $x>x_{0}$ ". The letters $C, K$, etc. will denote different constants in different contexts.

2. Preliminary lemmas and definitions. Assume (as will be shown in the corollary following Lemma 7 of $\S 4$ ) that $L>0$. Then if $f$ is in $\mathscr{M}$ and $a$ is a real number, define $\Lambda(=\Lambda(a))$ and $\chi(=\chi(a))$ by

$$
\begin{aligned}
e^{\Lambda a} & =\limsup _{x \rightarrow \infty}\{f(x+a) / f(x)\} \\
& =\limsup _{x \rightarrow \infty}\{g(x+a) / g(x)\}, \\
e^{x a} & =\liminf _{x \rightarrow \infty}\{f(x+a) / f(x)\} \\
& =\liminf _{x \rightarrow \infty}\{g(x+a) / g(x)\},
\end{aligned}
$$

where, as usual, $g(x)=\int k(x-y) f(y) d y$. The latter equalities in (2.1) and (2.2) are valid since $g / f \rightarrow L>0$. If $f$ is in $\mathscr{M}^{\prime}$, then $\chi>-\infty$ for every real $a$, and Lemma 8 yields that we may also assume $\Lambda<\infty$.

Both $\Lambda$ and $\chi$ depend on the choice of $a$; we compare them with $\lambda$, the (real) exponential type of $f$ :

$$
\begin{aligned}
\lambda & =\limsup _{x \rightarrow \infty}\{\log f(x) / x\} \\
& =\limsup _{x \rightarrow \infty}\{\log g(x) / x\} .
\end{aligned}
$$

LEMMA 1. For every $a>0, \chi(=\chi(a)) \leqq \lambda \leqq \Lambda(=\Lambda(a))$.

Proof. We show only that $\lambda \leqq \Lambda$, as the other inequality is more readily seen. If $\lambda=\Lambda+2 \varepsilon(\varepsilon>0)$, then there would exist $r_{0}$ with $g(r+a) / g(r)<e^{(\lambda-\varepsilon) a}\left(r>r_{0}\right)$. In particular, this is true when $r_{0} \leqq r \leqq r_{0}+a$. Hence, for every natural number $n$,

$$
g(r+n a) / g(r)<e^{n(\lambda-\varepsilon) a}, \quad r_{0}<r \leqq r_{0}+a .
$$

Choose $r_{1}, r_{0}<r_{1}<r_{0}+a$, with the property that

$$
g\left(r_{1}\right) \geqq 1 / 2 \sup _{r_{0}<t \leqq r_{0}+a} g(t)
$$

(if $g$ were assumed continuous, the $1 / 2$ could be replaced by 1 ); since $g$ is also in $\mathscr{M}$, we may assume that $r_{0}$ is sufficiently large to ensure that $g\left(r_{1}\right)>0$.

Now if $y>r_{0}$, find integer $n$ such that $r_{0}+n a<y \leqq r_{0}+(n+1) a$, and then $r^{*}$ with $y=r^{*}+n a$. Then, from (2.4)

$$
\begin{aligned}
g(y) & <g\left(r^{*}\right) e^{(\lambda-\varepsilon)\left(y-r^{*}\right)} \\
& \leqq 2 g\left(r_{1}\right) e^{(\lambda-\varepsilon)\left(y-r_{1}\right)} e^{|\lambda-\varepsilon| a} \\
& =K e^{(\lambda-\varepsilon) y}
\end{aligned}
$$


Taking logarithms of both sides and letting $y \rightarrow \infty$ leads to the definition (2.3) being contradicted.

This lemma establishes the plan of attack: the goal is to show that

$$
\chi=\lambda=\Lambda \text { for each } a
$$

these equalities are equivalent to (1.8) and (1.9).

It is convenient to insert a lemma to be used in the asymptotic computations that will be employed. The validity of this lemma provides the major reason for our exclusive concern with class $\mathscr{M}$. The proof is a trivial application of (1.1), (1.2) and the integrability of $k$.

LeMma 2. Let $f$ be in $\mathscr{M}$. Then for any real $R$ and $a$,

$$
\int_{-\infty}^{R} k(x-y) f(y+a) d y=o\{f(x)\} \quad(x \rightarrow \infty)
$$

3. Exponential peaks and proof of Theorem 1. The asymptotic computations necessary for our proofs are greatly simplified by isolating arbitrarily large intervals in which the function $f$ which is assumed to satisfy (1.7) is comparable to an exponential function. A similar technique has been exploited in several recent papers devoted to the Nevanlinna theory of meromorphic functions (e.g. [1]).

Definition. Let $\alpha$ and $a$ be real numbers. A sequence $\left\{r_{m}\right\}_{m=1}^{\infty}$ tending to $\infty$ is a sequence of upper $\alpha$-exponential peaks for $f$ if

$$
f(t) \leqq f\left(r_{m}\right) e^{\alpha\left(t-r_{m}\right)}(1+o(1))
$$

uniformly as $t \rightarrow \infty$ in the interval $-m a+r_{m} \leqq t \leqq m a+r_{m}$. Dually, a sequence $\left\{s_{m}\right\}_{m=1}^{\infty}$ tending to infinity is a sequence of lower $\alpha$-exponential peaks for $f$ if

$$
f(t) \geqq f\left(s_{m}\right) e^{\alpha\left(t-s_{m}\right)}(1+o(1))
$$

uniformly as $t \rightarrow \infty$ in $-m a+s_{m} \leqq t \leqq m a+s_{m}$.

THEOREM 2. Let $a$ be any real number and let $\Lambda=\Lambda(a)$ and $\chi=\chi(a)$, with the notation of (2.1) and (2.2). Then if $\alpha$ satisfies

$$
\chi \leqq \alpha \leqq \Lambda,
$$

there exist both upper and lower $\alpha$-exponential peaks for $f$.

The proof of this theorem occupies the major portion of this paper, and is deferred to $\$ \$-7$; we assume its validity for the remainder of this section. It is in its proof-particularly in the lemma of $\S 6$ - that the strict positivity of $k$ will be needed.

Now recall the number $\rho$ determined by (1.5).

Lemma 3. For every $a, \Lambda(=\Lambda(a))<\rho$. 
Proof. Let $\left\{s_{m}\right\}$ be lower $\Lambda$-exponential peaks; these exist from Theorem 2 . Then, from (3.2), with $\alpha=\Lambda$ and the nonnegativity of all functions considered, it follows that

$$
\begin{aligned}
g\left(s_{m}\right) & \geqq \int_{s_{m}-m a}^{s_{m}+m a} k\left(s_{m}-y\right) f(y) d y \\
& \geqq\{1+o(1)\} f\left(s_{m}\right) \int_{s_{m}-m a}^{s_{m}+m a} k\left(s_{m}-y\right) e^{-\Lambda\left(s_{m}-y\right)} d y \\
& =\{1+o(1)\} f\left(s_{m}\right)\left\{\int_{-m a}^{m a} k(t) e^{-\Lambda t} d t\right\} .
\end{aligned}
$$

Divide both sides of the inequality by $f\left(s_{m}\right)$, and let $m \rightarrow \infty$. Since $g / f \rightarrow L$ as $x \rightarrow \infty$,

$$
L \geqq \int_{-\infty}^{\infty} k(t) e^{-\Lambda t} d t
$$

in particular, $\mathscr{L} k(\Lambda)$ exists. Assumption (1.5) rules out the possibility that $\Lambda=\rho$. If $\Lambda$ were greater than $\rho$, this would contradict the fact that the domain of convergence of a bilateral Laplace transform is a full vertical strip of the complex plane.

The bound on $\Lambda$ just established leads at once to

LEMMA 4. Let $\left\{x_{m}\right\}$ be any sequence tending to infinity, and let $\eta$ be subject only to

$$
\Lambda<\eta<\rho,
$$

where $\Lambda=\Lambda(a)$ for some value of $a>0$. Then there are constants $C$ and $m_{0}$, such that

$$
f(y) \leqq C f\left(x_{m}\right) e^{\eta\left(y-x_{m}\right)} \quad\left(m>m_{0}\right) .
$$

Proof. It follows from (1.3) that for any real value of $a$ there are constants $K$ and $x_{1}$ with

$$
K \inf f(t)>f(x),
$$

whenever $x_{1}<x \leqq t \leqq x+a$. Since $\eta>\Lambda$, there is an $x_{0}\left(>x_{1}\right)$ such that

$$
f(x+a) / f(x)<e^{n a} \quad\left(x>x_{0}\right) .
$$

Now if $y \geqq x>x_{0}$, choose $x^{*}$ in the interval $(x, x+a]$ to satisfy $y-m a=x^{*}$, where $m$ is a natural number. Then, iteration of (3.7) leads to $f(y)<e^{n m a} f\left(x^{*}\right)$, which, upon taking (3.6) into account, becomes

$$
\begin{aligned}
f(y) & <e^{\eta\left(y-x^{*}\right)} K f(x) \\
& <C e^{\eta(y-x)} f(x) .
\end{aligned}
$$

Lemma 5. Let $\left\{x_{m}\right\} \rightarrow \infty$. Then

$$
\int_{x_{m}+m a}^{\infty} k\left(x_{m}-y\right) f(y) d y=o\left(f\left(x_{m}\right)\right)
$$


Proof. Otherwise, for some $\varepsilon>0$, there would be arbitrarily large values of $m$ for which

$$
\begin{aligned}
\varepsilon f\left(x_{m}\right) & <\int_{x_{m}+m a}^{\infty} k\left(x_{m}-y\right) f(y) d y \\
& <C f\left(x_{m}\right) \int_{-\infty}^{-m a} k(t) e^{-n t} d t ;
\end{aligned}
$$

we are using the estimate derived in Lemma 4 and an elementary change of variables. Since $\mathscr{L} k(\eta)$ exists,

$$
\int_{-\infty}^{-m a} k(t) e^{-\eta t} d t<\varepsilon / 2 C \quad\left(m>m_{1}\right)
$$

which, when substituted in (3.8) leads to a contradiction.

A similar result holds for the left "tail."

Lemma 6. Let $\left\{x_{m}\right\} \rightarrow \infty$. Then

$$
\int_{-\infty}^{x_{m}-m a} k\left(x_{m}-y\right) f(y) d y=o\left\{f\left(x_{m}\right)\right\}
$$

Proof. The argument is similar to that given in Lemmas 3-5. By considering lower $\chi$-exponential peaks, it follows that $\mathscr{L} k(\chi)$ exists, so, using the notation of (1.5), $-\sigma<\chi$. Then if $\tau$ is any number with $-\sigma<\tau<\chi$, and $R<y<x$

$$
f(y)<f(x) e^{-\tau(x-y)}
$$

which when combined with Lemma 2 gives the lemma.

Consider now $\mathscr{L} k$, the bilateral Laplace transform of $k$.

THEOREM 3. Let $\alpha$ be any real number satisfying $\chi \leqq \alpha \leqq \Lambda$. Then $\mathscr{L} k(\alpha)=L$.

Proof. Let $\left\{x_{m}\right\}$ be upper $\alpha$-exponential peaks, and write

$$
\begin{aligned}
g\left(x_{m}\right)= & \int_{-\infty}^{\infty} k\left(x_{m}-y\right) f(y) d y \\
= & \int_{-\infty}^{x_{m}-m a} k\left(x_{m}-y\right) f(y) d y+\int_{x_{m}-m a}^{x_{m}+m a} k\left(x_{m}-y\right) f(y) d y \\
& +\int_{x_{m}+m a}^{\infty} k\left(x_{m}-y\right) f(y) d y .
\end{aligned}
$$

Lemmas 5 and 6 imply that the first and third terms on the right side of this equality are $o\left(g\left(x_{m}\right)\right)$. Thus, from (3.1),

$$
\begin{aligned}
g\left(x_{m}\right) & =o\left(g\left(x_{m}\right)\right)+\int_{x_{m}-m a}^{x_{m}+m a} k\left(x_{m}-y\right) f(y) d y \\
& <o\left(g\left(x_{m}\right)\right)+f\left(x_{m}\right) \int_{x_{m}-m a}^{x_{m}+m a} e^{-\alpha\left(x_{m}-y\right)} k\left(x_{m}-y\right) d y \\
& =o\left(g\left(x_{m}\right)\right)+f\left(x_{m}\right) \int_{-m a}^{m a} e^{-\alpha t} k(t) d t .
\end{aligned}
$$


Divide both sides by $f\left(x_{m}\right)$ and let $m \rightarrow \infty$. Since $g / f \rightarrow L, L \leqq \mathscr{L} k(\alpha)$, and the reverse inequality follows exactly as in the proof of (3.4), upon replacing $\Lambda$ by $\alpha$.

Proof of Theorem 1. Suppose for some $a, \chi(=\chi(a))<\Lambda(=\Lambda(a))$. Then for all real $\alpha$ in the interval $\chi \leqq \alpha \leqq \Lambda, \mathscr{L} k(\alpha)=L$. But $\mathscr{L} k(s)$ is an analytic function of $s$ in the strip of convergence. Hence,

$$
\mathscr{L} k(s)=\int_{-\infty}^{\infty} e^{-s t} k(t) d t \equiv L, \quad 0<\operatorname{Re}(s)<\rho .
$$

Differentiation under the integral sign is permitted, and so

$$
(\mathscr{L} k)^{\prime}(s)=-\int_{-\infty}^{\infty} e^{-s t} t k(t) d t \equiv 0
$$

in the strip of convergence. This cannot happen unless $k$ is a null function [10], and this possibility is explicitly ruled out by (1.6). Thus $\chi=\Lambda=\lambda$ for every $a$; this is merely (2.5), so Theorem 1 is proved.

4. This and the next three sections are devoted to the proof of Theorem 2 . In addition, it will be shown that $L>0$, where $L$ is determined by (1.7).

The assumption (1.3) that there exist $a_{0}, x_{0}$ such that

$$
f(t) \mid f(x)>\varepsilon, \quad x_{0}<x \leqq t \leqq x+a_{0},
$$

for class $\mathscr{M}^{\prime}$ may seem one-sided. We first show that if $f$ is in $\mathscr{H}^{\prime}$ and $g(x) / f(x) \rightarrow L$, then $L>0$ and (4.1) is equivalent to the existence of an $\eta(\varepsilon)>0$, given $\varepsilon>0$, with

$$
f(t) / f(x)<1+\varepsilon, \quad x_{0}<x \leqq t \leqq x+\eta(\varepsilon) .
$$

LEMMA 7. If $f$ is in $\mathscr{M}^{\prime}$, then there are constants $C(>0)$ and $\gamma(>0)$ with

$$
f(y) \geqq C e^{-y(y-x)} f(x), \quad y \geqq x>x_{0} .
$$

Proof. Choose $\varepsilon, 0<\varepsilon<1$, and $a_{0}$ as in (1.3), and define $\gamma$ by $\varepsilon=e^{-\gamma a_{0}}$. If $n$ is a positive integer and $x+n a_{0} \leqq y<x+(n+1) a_{0}$, then from

$$
\frac{f(y)}{f(x)}=\frac{f\left(x+a_{0}\right)}{f(x)} \cdot \frac{f\left(x+2 a_{0}\right)}{f\left(x+a_{0}\right)} \cdots \frac{f(y)}{f\left(x+n a_{0}\right)}
$$

follows that

$$
f(y) \geqq e^{-|y| a_{0}} e^{-\gamma(y-x)} f(x)
$$

We are now able to settle an issue left unresolved at the beginning of $\$ 2$ :

COROllary. If $f$ is in $\mathscr{M}^{\prime}, k(t)$ satisfies (1.4), (1.5), and (1.6), and

$$
\int_{-\infty}^{\infty} k(x-y) f(y) d y=\{L+o(1)\} f(x), \quad(x \rightarrow \infty),
$$

then $L>0$. 
Proof. Indeed, using the last lemma, if $a>0$ and $x>x_{0}$,

$$
\begin{aligned}
g(x) & =\int_{-\infty}^{\infty} k(x-y) f(y) d y \\
& >\int_{x}^{\infty} k(x-y) f(y) d y \\
& >C f(x) \int_{-\infty}^{0} k(t) e^{-\gamma t} d t \\
& =C \alpha f(x)
\end{aligned}
$$

thus, $L \geqq C \alpha$.

A weak form of (4.2) may now be established:

LEMMA 8. Let the assumptions of Theorem 1 be satisfied. Then for each $a>0$, there are numbers $K(=K(a))$ and $x_{0}\left(=x_{0}(a)\right)$ such that

$$
\begin{array}{ll}
f(t) / f(x)<K, & x_{0}<x \leqq t \leqq x+a, \\
g(t) / g(x)<K, & x_{0}<x \leqq t \leqq x+a .
\end{array}
$$

Proof. It is clear from the last corollary that (4.4) and (4.5) each imply the other. Choose any $t$ in the interval $(x, x+a)$. Then, making use of Lemma 7, and the positivity of all functions considered,

$$
\begin{aligned}
g(x) & >\int_{t}^{\infty} k(x-y) f(y) d y \\
& >C f(t) \int_{-\infty}^{-a} e^{-r y} k(y) d y \\
& >(C \alpha / 2 L) g(t),
\end{aligned}
$$

with $\alpha>0$, in view of (1.6). Thus, when $x>x_{0}, g(t) / g(x)<(C \alpha) / 2 L$.

5. Proof of (4.2). For each $a>0$, set

$$
M(a ; f)=\limsup _{x \rightarrow \infty}\left\{\sup _{x \leqq t \leqq x+a} f(t) / f(x)\right\} .
$$

(Note from $(2.1)$ that $M(a ; f) \geqq e^{\Lambda a}$.) Clearly $M(a ; f)=M(a ; g) \geqq 1$, and the last lemma ensures that $M(a ; f)<\infty$. As $a$ decreases to zero, $M(a ; f)$ also decreases. Hence $M=\lim _{a \rightarrow 0+} M(a ; f)=\lim _{a \rightarrow 0+} M(a ; g)$ exists, and (4.2) asserts that $M=1$.

If, in fact, $M$ were greater than 1 , then there would exist sequences $\left\{x_{n}\right\} \rightarrow \infty$ and $\left\{t_{n}\right\} \downarrow 0$ with

$$
g\left(x_{n}+t_{n}\right) / g\left(x_{n}\right) \rightarrow M=1+H, \quad H>0 .
$$


Choose $\gamma>0$ so that if $E$ is any measurable subset of $(0,1)$ of measure $\geqq \frac{1}{2}$,

$$
\int_{E} k(t) d t>2 L \gamma
$$

and then determine $h(>0)$ by

$$
h=(1+H)-(1+H)^{1 / 2} .
$$

Finally, since $f$ is in $\mathscr{M}^{\prime}$, there exist $\varepsilon>0$ and $a_{0}>0$ with

$$
f(y) / f(x)>\varepsilon
$$

if $x_{0} \leqq x \leqq y \leqq x+a_{0}$.

For any $\xi>0$, and $a, 0<a<a_{0}$, the inequalities

$$
\begin{aligned}
& f(t) / f(x)<M+\xi, \\
& g(t) / g(x)<M+\xi
\end{aligned}
$$

hold when $R<x \leqq t \leqq x+a$ for some $R$. Choose $\xi$ to satisfy

$$
0<\xi<h \varepsilon \gamma,
$$

where the constants on the right side of (5.7) are determined by (5.3), (5.4) and (5.5). By appropriate relabelling if necessary, assume $x_{n}>R$ and $t_{n}<\frac{1}{2} a$ $(n=1,2, \ldots)$. Finally, define

$$
E_{n}(\xi)=\left\{y ;-1 \leqq y-x_{n} \leqq 0, f\left(y+t_{n}\right) \leqq(M+\xi)^{1 / 2} f(y)\right\}
$$

Since (5.6) implies

$$
\begin{aligned}
M+\xi & \geqq f\left(y+2 t_{n}\right) / f(y) \\
& =\left\{f\left(y+2 t_{n}\right) / f\left(y+t_{n}\right)\right\}\left\{f\left(y+t_{n}\right) / f(y)\right\},
\end{aligned}
$$

it follows that if $-1 \leqq y-x_{n} \leqq 0$, either $y$ or $y+t_{n}$ is in $E_{n}(\xi)$. Thus, the measure of $E_{n}(\xi)$ is at least $1 / 2$.

However,

$$
\begin{aligned}
\dot{g}\left(x_{n}+t_{n}\right) & =\int_{-\infty}^{\infty} k\left(x_{n}+t_{n}-y\right) f(y) d y \\
& =\int_{-\infty}^{\infty} k\left(x_{n}-y\right) f\left(y+t_{n}\right) d y,
\end{aligned}
$$

so (5.6) and Lemma 2 lead to

$$
\begin{aligned}
g\left(x_{n}+t_{n}\right) \leqq & \int_{-\infty}^{R} k\left(x_{n}-y\right) f\left(y+t_{n}\right) d y+(M+\xi) \int_{-\infty}^{\infty} k\left(x_{n}-y\right) f(y) d y \\
& +\int_{E_{n}(\xi)} k\left(x_{n}-y\right)\left\{f\left(y+t_{n}\right)-(M+\xi) f(y)\right\} d y \\
= & \{M+\xi+o(1)\} g\left(x_{n}\right)+\int_{E_{n}(\xi)} k\left(x_{n}-y\right)\left\{f\left(y+t_{n}\right)-(M+\xi) f(y)\right\} d y
\end{aligned}
$$


and this last term requires further attention. From the definition (5.8) of $E_{n}(\xi)$, it is negative, and since $E_{n}(\xi)$ has measure at least $1 / 2,(5.3),(5.4)$, and (5.5) show that

$$
\begin{aligned}
& \int_{E_{n}(\xi)} k\left(x_{n}-y\right)\left\{f\left(y+t_{n}\right)-(M+\xi) f(y)\right\} d y \\
& \leqq-h \int_{E_{n}(\xi)} k\left(x_{n}-y\right) f(y) d y \\
& \leqq-h \varepsilon f\left(x_{n}\right) \cdot 2 L \gamma \\
& \leqq-h \varepsilon \gamma g\left(x_{n}\right) \quad\left(n>n_{0}\right) .
\end{aligned}
$$

Upon incorporating this estimate in (5.9), it follows that

$$
g\left(x_{n}+t_{n}\right) \leqq\{M+\xi-h \varepsilon \gamma+o(1)\} g\left(x_{n}\right) \quad\left(n>n_{0}\right) .
$$

Now divide both sides of (5.10) by $g\left(x_{n}\right)$, and let $n \rightarrow \infty$. The choice of $\xi$ in (5.7) ensures that

$$
\limsup _{x \rightarrow \infty} g\left(x_{n}+t_{n}\right) / g\left(x_{n}\right)<M-\eta
$$

for some $\eta>0$, and this contradicts (5.2).

RemarK. Let $\mathscr{M}^{\prime \prime}$ be the subclass of $\mathscr{M}$ consisting of those functions for which there exist $K, a_{0}(>0)$ and $x_{0}$ with

$$
f(t) / f(x)<K<\infty \quad\left(x>x_{0}\right)
$$

when $x \leqq t \leqq x+a_{0}$, and consider the statement of Theorem 1 with $f$ assumed in $\mathscr{M}^{\prime \prime}$ instead of $\mathscr{M}^{\prime}$. Inequality (4.2) makes this seem a weaker hypothesis, but the arguments of $\$ \$ 4$ and 5 easily generalize to show that for $\varepsilon>0$, there would exist an $a>0$ with

$$
f(t) / f(x)>1+\varepsilon, \quad x_{0}<x \leqq t \leqq x+a .
$$

Thus, if $f$ is in $\mathscr{M}, k$ satisfies (1.4)-(1.6) and $g(x) / f(x) \rightarrow L$, conditions (4.1) and (4.2) are equivalent.

6. The main lemma. Let $a(>0)$ be fixed in the present section, and define $N(=N(a))$ and $\nu(=\nu(a))$ by

$$
\begin{gathered}
N=\limsup _{x \rightarrow \infty} g(x+a) / g(x), \\
\nu=\liminf _{x \rightarrow \infty} g(x+a) / g(x) .
\end{gathered}
$$

Clearly, from (4.1), (5.1) and Lemma 8, $-\infty<\nu \leqq N \leqq M(a ; g)<\infty$. (In the notation of (2.1) and (2.2), $N=e^{\Lambda a}$ and $\nu=e^{x a}$.) The next lemma parallels Lemma 1 of [2].

Lemma 9. Consider sequences $\left\{x_{k}\right\}$ and $\left\{y_{k}\right\}$ tending to infinity with

$$
\begin{aligned}
& g\left(x_{k}+a\right) / g\left(x_{k}\right) \rightarrow N, \\
& g\left(y_{k}+a\right) / g\left(y_{k}\right) \rightarrow \nu .
\end{aligned}
$$


I. Then, given $A(>0)$ and $\varepsilon(>0)$,

$$
g(s+a) / g(s)>N-\varepsilon
$$

whenever $x_{k}-A \leqq s \leqq x_{k}+A, k>K_{0}(A, \varepsilon, a)$.

II. Similarly,

$$
g(s+a) / g(s)<\nu+\varepsilon
$$

whenever

$$
y_{k}-A \leqq s \leqq y_{k}+A, \quad k>K_{0}(A, \varepsilon, a) .
$$

Proof. Only I will be discussed. If it were false, then for some $\eta>0$, there would be arbitrarily large values of $k$ with $g(s+a)<(N-3 \eta) g(s)$ for $s=s_{k}$ in the interval $\left[x_{k}-A, x_{k}+A\right]$. Discard all values of $k$ for which such an $s_{k}$ fails to exist, and renumber the remaining $x_{k}$ (and corresponding $s_{k}$ ) from 1 to $\infty$.

Now select $\theta, 0<\theta<\frac{1}{2}$, such that

$$
(N-3 \eta)(1+\theta)(1-\theta)^{-2}<N-2 \eta,
$$

and next, in accord with (4.2) and (5.12), choose $\sigma>0$ so that $g$ satisfies

$$
g(x)(1-\theta)<g(t)<(1+\theta) g(x)
$$

whenever $x_{0}<x \leqq t \leqq x+\sigma$. Then if $s_{k} \leqq y \leqq s_{k}+\sigma$,

$$
\begin{aligned}
\frac{g(y+a)}{g(y)} & =\frac{g\left(s_{k}+a\right)}{g\left(s_{k}\right)} \cdot \frac{g(y+a)}{g\left(s_{k}+a\right)} \cdot \frac{g\left(s_{k}\right)}{g(y)} \\
& \leqq(1-\theta)^{-2} \frac{g\left(s_{k}+a\right)}{g\left(s_{k}\right)} \cdot \frac{g\left(s_{k}+a+\sigma\right)}{g\left(s_{k}+a\right)} \\
& \leqq(N-3 \eta)(1+\theta)(1-\theta)^{-2} \\
& <N-2 \eta
\end{aligned}
$$

if $k$ is sufficiently large.

Since, by (1.6), $k>0$ a.e., there is a $\gamma>0$ such that if $E$ is an interval of length $\sigma$ contained in the interval $-A-\sigma \leqq t \leqq A$,

$$
\int_{E} k(t) d t>\gamma
$$

Finally, we find $K>0$ such that if $-A \leqq t \leqq A$ and $y>y_{0}$,

$$
g(y+t)>K g(y)
$$

this is possible in view of (4.2) and (5.12).

Suppose $\xi$ is determined by

$$
0<\xi<(1 / 2 L) \eta \gamma K .
$$


Then there is an $R=R(\xi)$ with the property that when $t>R$,

$$
g(t+a)<(N+\xi) g(t)
$$

and

$$
f(t+a)<(N+\xi) f(t) .
$$

In view of (6.3) and the Tauberian assumption (1.7), we may assume that $R$ is so large that

$$
f(t+a)<(N-\eta) f(t)
$$

provided

$$
R<x_{k}-A \leqq s_{k} \leqq t \leqq s_{k}+\sigma \leqq x_{k}+A+\sigma .
$$

As we saw in $\S 5$,

$$
g\left(x_{k}+a\right)=\int_{-\infty}^{\infty} k\left(x_{k}-y\right) f(y+a) d y
$$

so we deduce from (6.8) that

$$
\begin{gathered}
\int_{R}^{\infty} k\left(x_{k}-y\right) f(y+a) d y<\{N+\xi\} \int_{R}^{\infty} k\left(x_{k}-y\right) f(y) d y \\
+\int_{s_{k}}^{s_{k}+\sigma} k\left(x_{k}-y\right)\{f(y+a)-(N+\xi) f(y)\} d y,
\end{gathered}
$$

and that last integral invites further attention. It is negative and, in view of (6.4) and (6.9), does not exceed

$$
-(\xi+\eta) \int_{s_{k}}^{s_{k}+\sigma} k\left(x_{k}-y\right) f(y) d y \leqq-(\xi+\eta)\left\{\inf _{0 \leqq u \leqq \sigma} f\left(s_{k}+u\right)\right\} \int_{s_{k}}^{s_{k}+\sigma} k\left(x_{k}-y\right) d y
$$

$$
\begin{aligned}
& \leqq-(\xi+\eta) 3 / 4 f\left(s^{*}\right) \int_{x_{k}-s_{k}-\sigma}^{x_{k}-s_{k}} k(t) d t \\
& \leqq-(\xi+\eta)\left(g\left(s^{*}\right) / 2 L\right) \gamma
\end{aligned}
$$

when $k$ is large; $s^{*}$ is a point in $\left[s_{k}, s_{k}+\sigma\right]$ for which $f\left(s^{*}\right) \leqq 4 / 3 \inf _{0 \leqq u \leqq \sigma} f\left(s_{k}+u\right)$. Inequality (6.5) implies that $g\left(s^{*}\right)>K g\left(x_{k}\right)$. We incorporate this estimate of $g\left(s^{*}\right)$ in (6.12). Thus,

$$
\int_{R}^{\infty} k\left(x_{k}-y\right) f(y+a) d y \leqq\{N+\xi\} \int_{R}^{\infty} k\left(x_{k}-y\right) f(y) d y-(1 / 2 L)(\xi+\eta) K \gamma g\left(x_{k}\right),
$$

and we use this in (6.10). Upon incorporating Lemma 2 into the above calculations, it follows that

$$
\begin{aligned}
g\left(x_{k}+a\right) & \leqq \int_{-\infty}^{R} k\left(x_{k}-y\right) f(y) d y+(N+\xi) g\left(x_{k}\right)-(1 / 2 L)(\xi+\eta) K \gamma g\left(x_{k}\right) \\
& \leqq\{o(1)+(N+\xi)-(1 / 2 L) \eta \gamma K\} g\left(x_{k}\right)
\end{aligned}
$$


Now divide both sides of (6.13) by $g\left(x_{k}\right)$, and let $k \rightarrow \infty$. Since $\xi$ was chosen to satisfy (6.6), assumption (6.1) is contradicted.

REMark. The result of this lemma holds also for $f$, since $g / f \rightarrow L>0$. Thus, given $A, \varepsilon>0$,

$$
f(s+a) / f(s)>N-\varepsilon
$$

whenever

$$
x_{k}-A<s \leqq x_{k}+A, \quad k>K_{0}(A, \varepsilon, a),
$$

and $f(s+a) / f(s)<\nu+\varepsilon$ whenever $y_{k}-A \leqq s \leqq y_{k}+A, k>K_{0}(A, \varepsilon, a)$.

7. Exponential peaks: Proof of Theorem 2. We return to the definitions of exponential peaks of $\S 3$ and of the constants $\chi(=\chi(a))$ and $\Lambda(=\Lambda(a))$ of $\S 2$. There are three cases to be considered.

CASE 1. $\alpha=\Lambda$. First choose a sequence $\left\{u_{k}\right\}$ tending to infinity with

$$
\lim _{k \rightarrow 0} f\left(u_{k}+a\right) / f\left(u_{k}\right)=e^{\Lambda a} .
$$

It follows from (6.14) and (6.15) that for each integer $m$,

$$
e^{\Lambda a-1 /(m+1)^{2}}<f(t+a) / f(t)<e^{\Lambda a+1 /(m+1)^{2}}
$$

provided

$$
u_{k}-(m+1) a \leqq t \leqq u_{k}+(m+1) a, \quad k>k_{0} .
$$

Let $u_{m}^{\prime}$ be a value of $u_{m}$ large enough to imply $u_{m}^{\prime}-m a \geqq m$, and then determine $r_{m}$ and $s_{m}$ so that $u_{m}^{\prime} \leqq r_{m}, s_{m} \leqq u_{m}^{\prime}+a$ and

$$
\begin{aligned}
& (1+1 / m) \cdot f\left(r_{m}\right) e^{-\Delta r_{m}} \geqq \sup f(t) e^{-\Lambda t}, \\
& (1-1 / m) \cdot f\left(s_{m}\right) e^{-\Lambda s_{m}} \leqq \inf f(t) e^{-\Lambda t},
\end{aligned}
$$

for $u_{m}^{\prime} \leqq t \leqq u_{m}^{\prime}+a$. (If $f$ were known to be continuous, the factors $(1 \pm 1 / m)$ could be replaced by 1.) We shall prove that the $\left\{r_{m}\right\}$ are upper $\Lambda$-exponential peaks; the reader will be able to adapt these arguments suitably to show that the $\left\{s_{m}\right\}$ are lower $\Lambda$-exponential peaks.

If $-m a+r_{m} \leqq t \leqq r_{m}+m a, t$ will satisfy

$$
-(m+1) a+u_{m}^{\prime} \leqq t \leqq(m+1) a+u_{m}^{\prime} .
$$

Suppose first that

$$
u_{m}^{\prime} \leqq t \leqq r_{m}+m a
$$

thus,

where

$$
t=u_{m}^{\prime}+(\gamma+n) a
$$

$$
0 \leqq n \leqq m, \quad 0 \leqq \gamma \leqq 1
$$


The second inequality in (7.1) implies that for $j=1,2, \ldots, n$,

$$
f\left(u_{m}^{\prime}+(\gamma+j-1) a\right) e^{\Lambda a+1 /(m+1)^{2}}>f\left(u_{m}^{\prime}+(\gamma+j) a\right),
$$

so, upon combining these $n$ inequalities,

$$
\begin{aligned}
f(t) & \leqq f\left(u_{m}^{\prime}+\gamma a\right) e^{\Lambda n a+n /(m+1)^{2}} \\
& \leqq f\left(u_{m}^{\prime}+\gamma a\right) e^{\Lambda n a+1 /(m+1)} .
\end{aligned}
$$

If (7.2) is incorporated with (7.7), it follows that

$$
f(t) \leqq f\left(r_{m}\right) e^{\Lambda\left(t-r_{m}\right)}\left\{e^{1 /(m+1)}(1+1 / m)\right\}
$$

which proves (3.1) if $t$ satisfies (7.5). If

$$
r_{m}-m a \leqq t \leqq u_{m}^{\prime},
$$

we write

$$
t=u_{m}^{\prime}-(\gamma+n) a
$$

where (7.6) holds. The left inequality of (7.1) shows that for $j=1,2, \ldots,(n+1)$,

$$
f\left(u_{m}^{\prime}+(1-\gamma-j) a\right) e^{\Lambda a-1 /(m+1)^{2}}<f\left(u_{m}^{\prime}+(2-\gamma-j) a\right),
$$

so combining these inequalities,

$$
f(t)<f\left(u_{m}^{\prime}+(1-\gamma) a\right) e^{-(n+1) \Delta a-1 /(m+1)} .
$$

After (7.2) is taken into account, this becomes

$$
f(t)<f\left(r_{m}\right) e^{\Lambda\left(t-r_{m}\right)}\left\{e^{1 /(m+1)}(1+1 / m)\right\}
$$

when $t$ satisfies (7.8). Thus, Theorem 2 is proved for the special case $\alpha=\Lambda$.

CASE 2. $\alpha=\chi$. A development dual to that of case 1 produces the required peaks.

CASE 3. $\chi<\alpha<\Lambda$. We first produce the upper peaks. Let $\left\{V_{m}\right\}$ be upper $\Lambda$ exponential peaks and $\left\{T_{m}\right\}$ be upper $\chi$-exponential peaks. By suppressing some of these, it is possible to assume that $T_{1}<V_{1}<T_{2}<V_{2} \cdots$.

Consider the auxiliary function $F(x)=f(x) e^{-\alpha x}$. For each natural number $m$, choose $R_{m}$ with the property that $R_{m}$ is in $I_{m}$ and

$$
F\left(R_{m}\right)(1+1 / m) \geqq \sup _{I_{m}} F(t) ;
$$

we are denoting by $I_{m}$ the interval $\left[V_{m}-m a, T_{m+1}+m a\right]$. The desired upper peaks $\left\{r_{m}\right\}$ may now be selected:

$$
\begin{aligned}
& r_{m}=V_{m} \quad \text { if } \quad V_{m}-m a \leqq R_{m} \leqq V_{m} \\
& =R_{m} \quad \text { if } \quad V_{m}<R_{m}<T_{m+1} \\
& =T_{m+1} \text { if } T_{m+1} \leqq R_{m} \leqq T_{m+1}+m a .
\end{aligned}
$$

Inequality (3.1) is certainly satisfied when $r_{m}=R_{m}$. Suppose, however, that $V_{m}-m a \leqq R_{m} \leqq V_{m}$. It is only necessary to prove that

$$
F\left(r_{m}\right) \geqq F\left(R_{m}\right)(1+o(1)) .
$$


Since the $V_{m}$ are upper $\Lambda$-exponential peaks,

$$
f\left(R_{m}\right) \leqq f\left(V_{m}\right) e^{\Lambda\left(R_{m}-V_{m}\right)}(1+o(1)),
$$

so, noting that $R_{m} \leqq V_{m}, \Lambda \geqq 0$ and $r_{m}=V_{m}$, it follows that

$$
\begin{aligned}
F\left(r_{m}\right) & =f\left(r_{m}\right) e^{-\alpha r_{m}} \\
& =f\left(V_{m}\right) e^{-\alpha V_{m}} \\
& \geqq f\left(R_{m}\right) e^{\Lambda\left(V_{m}-R_{m}\right)-\alpha V_{m}(1+o(1))} \\
& \geqq f\left(R_{m}\right) e^{\alpha\left(V_{m}-R_{m}\right)-\alpha V_{m}(1+o(1))} \\
& =f\left(R_{m}\right) e^{-\alpha R_{m}(1+o(1))} \\
& =F\left(R_{m}\right)(1+o(1)) .
\end{aligned}
$$

A similar argument applies when $T_{m+1} \leqq R_{m} \leqq T_{m+1}+m a$, in which case $r_{m}=R_{m}$, so the $\left\{r_{k}\right\}$ are upper $\alpha$-exponential peaks.

The lower peaks are constructed analogously, using the intervals $J_{m}=\left[t_{m}-m a\right.$, $\left.v_{m}+m a\right]$ where $t_{1}<v_{1}<t_{2}<v_{2} \cdots$, the $\left\{t_{m}\right\}$ are lower $\chi$-exponential peaks, and the $\left\{v_{m}\right\}$ are lower $\Lambda$-exponential peaks. We omit the details.

8. A converse to Theorem 1. Let $k(x)$ be a nonnegative function whose bilateral Laplace transform exists when $-\delta<s<\rho \leqq \infty$, with $\delta, \rho>0$ (note that (1.6) is not needed). Let $\psi(x)$ be any nonnegative function with the property that

$$
\psi(x+a) / \psi(x) \rightarrow 1 \text { for every } a, x \rightarrow \infty .
$$

Next, define

$$
f(x)=e^{\lambda x} \psi(x)
$$

and assume that

$$
f(x) \text { is in } \mathscr{M}
$$

(thus (1.3) need not be assumed). Note that (8.1) implies that if $\eta<0, e^{n t} \psi(t) \rightarrow 0$, so if $f$ is to be in $\mathscr{M}$, it follows that $\lambda \geqq 0$.

THEOREM 4. Let $f$ satisfy (8.3) and (8.2) (where $\psi$ satisfies (8.1)). If $g$ is defined by

$$
g(x)=\int_{-\infty}^{\infty} k(x-y) f(y) d y,
$$

then as $x \rightarrow \infty, g(x) / f(x) \rightarrow L$, where

$$
L=\mathscr{L} k(\lambda)=\int_{-\infty}^{\infty} e^{-\lambda t} k(t) d t>0 .
$$

REMARK. If either $\lambda>0$ or $f$ is monotone increasing it is possible to drop the condition that $\mathscr{L} k(s)$ exist when $-\delta<s \leqq 0(\delta>0)$.

The proof of Theorem 4 depends on a property of a function $\psi(x)$ satisfying (8.1) that was discovered by Karamata [6] (for an independent proof, see Besicovitch's lemma in [5]). 
LEMMA 10 (KARAMATA). Let $\psi(x)$ be a real-valued function for which (8.1) holds. Then $\psi(x+a) / \psi(x) \rightarrow 1$ uniformly, as $x \rightarrow \infty$, in any interval $-A \leqq a \leqq A$.

Proof of Theorem 4. The theorem claims, after simple manipulation, that

$$
\int_{-\infty}^{\infty} k(x-y) e^{\lambda(y-x)}\{\psi(y) / \psi(x)-1\} d y<5 \varepsilon
$$

Let $A>0$, to be determined later, and partition the range of integration in (8.4) into $(x+A, \infty),(x-A, x+A)$, and $(-\infty, x-A)$, denoting these corresponding integrals by $I_{1}, I_{2}$, and $I_{3}$.

Consider first $I_{1}$. We find an $A_{0}$ with the property that

$$
\int_{x+A}^{\infty} k(x-y) e^{-\lambda(x-y)} d y<\varepsilon \quad\left(A>A_{0}, x>x_{0}\right)
$$

and

$$
\int_{x+A}^{\infty} k(x-y) e^{-\lambda(x-y)} \psi(y) a y<\varepsilon \psi(x) \quad\left(A>A_{0}, x>x_{0}\right) .
$$

Inequality (8.5) is immediate since $\mathscr{L} k(\lambda)$ exists. To see that an $A_{0}$ may be chosen to satisfy (8.6), choose $\eta>0$ to satisfy $\lambda+\eta \cdot \rho$, and then find $x_{0}$ sufficiently large so that

$$
\psi(x+u) / \psi(x)<e^{\eta}
$$

when $0 \leqq u \leqq 1$ and $x>x_{0}$; this follows from Lemma 6. An analogue of Lemma 4 yields that if $y \geqq x \geqq x_{0}, \psi(y)<C e^{\eta(y-x)} \psi(x)$, from which (8.6) follows at once.

For any fixed $A, I_{2}<\varepsilon$ if $x$ is sufficiently large since

$$
k(t) e^{-\lambda t}\{\psi(x-t) / \psi(x)-1\} \rightarrow 0
$$

as $x \rightarrow \infty$, dominated (for large $x$ ) by $2 k(t) e^{-\lambda t} \in L_{1}$.

We turn to $I_{3}$ and (after a change of variables) show that $A_{1}$ and $x_{0}$ exist with the property that $\int_{A}^{\infty} k(t) e^{-\lambda t} d t<\varepsilon\left(A>A_{1}\right)$ and

$$
\int_{A}^{\infty} k(t) e^{-\lambda t}\{\psi(x-t) / \psi(x)\} d t<\varepsilon \quad\left(A>A_{1}, x>x_{0}\right) .
$$

Only the proof of (8.8) need be given. Choose $\varepsilon^{*}, 0<\varepsilon^{*}<\lambda+\delta$ (recall that $\lambda \geqq 0$ ). An argument dual to that which led to Lemma 4 yields that there is an $x_{1}$ with $\psi(u)<C e^{\varepsilon^{*}(v-u)} \psi(v), x_{1}<u \leqq v$. Let $u=x-t$ and $v=x$; then the last inequality becomes

$$
\psi(x-t)<C e^{\varepsilon^{*}} \psi(x), \quad 0 \leqq t<x-x_{1} .
$$

Next, using (1.2), determine $x_{0}\left(>x_{1}\right)$ such that

$$
f(x)>\eta>0, \quad x>x_{0} .
$$


Finally, if $t>x-x_{0},(1.1)$ implies that

$$
f(x-t)<\mu\left(x_{0} ; f\right)<\infty .
$$

Then if $x>x_{0}$, estimates (8.9), (8.10) and (8.11) show that the integral in (8.8) is majorized by

$$
\begin{aligned}
C \int_{A}^{x-x_{0}} e^{-\left(\lambda-\varepsilon^{*}\right) t} k(t) d t+ & \frac{\mu\left(x_{0} ; f\right)}{f(x)} \int_{x-x_{0}}^{\infty} k(t) d t \\
& \leqq\left\{\mu\left(x_{0}\right) / \eta+C\right\}\left\{\int_{A}^{\infty} e^{-\left(\lambda-\varepsilon^{*}\right) t} k(t) d t+\int_{A}^{\infty} k(t) d t\right\}
\end{aligned}
$$

But both $k(t)$ and $e^{-\left(\lambda-\varepsilon^{*}\right) t} k(t)$ are in $L_{1}(0, \infty)$, and so (8.8) can be made as small as desired, when $x>x_{0}$, if $A$ is sufficiently large.

9. Some counterexamples. We give two examples to show that the conclusion of Theorem 1 need not follow from the condition

$$
\int_{-\infty}^{\infty} k(x-y) f(y) d y=\{L+o(1)\} f(x)
$$

if either $k$ or $f$ assumes both positive or negative values.

Let $h(t)(0 \leqq t \leqq 2)$ be a continuous function satisfying

$$
\begin{aligned}
h(1-u) & =h(1+u), \quad 0 \leqq u \leqq 1, \\
h(0) & =h(2)=0,
\end{aligned}
$$

and, given $\lambda>0$, define $k(t)$ for all values of $t$ by

$$
k(t)=C_{n} e^{\lambda t} h(t-2 n),
$$

when $2 n \leqq t \leqq 2 n+2, n=0, \pm 1, \pm 2, \ldots$. The $C_{n}$ are positive numbers chosen so that $k$ fulfills (1.4), (1.5) (for some $\rho>\lambda$ ).

Suppose we no longer insisted that $k$ be of constant sign. It is then easy to find an $h$ defined on $[0,2]$ which satisfies (9.1), (9.2), vanishes for only four values of $t$, and satisfies

$$
\int_{0}^{2} h(t) d t=\int_{0}^{2} h(t) \cos \pi t d t=\beta>0 .
$$

Let $f(t)=e^{\lambda t}(A+\sin \pi t)$, where $A>\lambda^{-1}(1+\pi)$; then $f$ is nonnegative and increasing, so in $\mathscr{M}$. Note, however, that $f$ is not of the form $e^{\lambda t} \psi(t)$, where $\psi(t)$ satisfies (1.3). However

$$
\begin{aligned}
g(x) & =\int_{-\infty}^{\infty} k(x-y) f(y) d y \\
& =\int_{-\infty}^{\infty} f(x-y) k(y) d y \\
& =\int_{-\infty}^{\infty} e^{\lambda(x-y)}\{A+\sin \pi x \cos \pi y-\cos \pi x \sin \pi y\} k(y) d y .
\end{aligned}
$$


Upon writing this last term as three individual integrals, and observing (9.1), (9.2) and (9.4), it follows readily that $g(x)=\left\{\beta \cdot \sum C_{n}\right\} f(x)$.

If $k$ is always to be nonnegative but $f$ is allowed to vary sign, consider $f(x)$ $=e^{\lambda x} \sin \pi x$, let $h$ be positive and satisfy (9.1) and (9.2), and define $k$ as in (9.3). Then, $g(x)=\left\{\beta \sum C_{n}\right\} f(x)$, although $f$ is not of the form $e^{\lambda x} \psi(x)$, where $\psi$ satisfies (1.3).

The same examples show that $k$ and $f$ may not vary sign if we are to draw the conclusion of Theorem 1 from the assumption that

$$
\int_{0}^{x} k(x-y) f(y) d y=\{L+o(1)\} f(x)
$$

which is considered in $\$ 11$.

10. An application of Theorem 1 to entire functions. Let $f$ be an entire function of the complex variable $z$, and let $M(r)=\max _{|z|=r}|f(z)|$. The order $\lambda$ of $f$ is defined by

$$
\lambda=\limsup _{r \rightarrow \infty} \frac{\log \log M(r)}{\log r}
$$

and we assume here that $0<\lambda<1$.

Finally, let $n(t)$ denote the number of zeros of $f$ in $|z| \leqq t$ (for convenience, we assume $f(0)=1$ ) and

$$
N(r)=\int_{0}^{t} \frac{n(t)}{t} d t
$$

Valiron [9] proved that if all the zeros of $f$ are real and negative, then either of

$$
n(r) \sim r^{\lambda} \psi(r) \quad(r \rightarrow \infty)
$$

or

$$
N(r) \sim \lambda^{-1} r^{\lambda} \psi(r) \quad(r \rightarrow \infty)
$$

where $\psi$ satisfies (3) are equivalent to

$$
\log M(r)=\log f(r) \sim\{\pi \csc \pi \lambda\} r^{\lambda} \psi(r) \quad(r \rightarrow \infty) .
$$

We have the complementary result, essentially due to Edrei and Fuchs [2]:

THEOREM 5. If $f$ is of order $\lambda, 0<\lambda<1$, with all zeros real and negative, then either of the conditions

$$
\log M(r) / n(r) \rightarrow L>0 \quad(r \rightarrow \infty)
$$

or

$$
\log M(r) / N(r) \rightarrow L / \lambda>0 \quad(r \rightarrow \infty)
$$

imply that

$$
\log M(r)=r^{\lambda} \psi(r)
$$


where for each fixed $\sigma>0$,

$$
\psi(\sigma r) / \psi(r) \rightarrow 1 \quad(r \rightarrow \infty) .
$$

Further, $\lambda$ is determined by

$$
L=\pi \csc \pi \lambda .
$$

Proof. We use the classical formula [8]

$$
\log M(r)=\log f(r)=r \int_{0}^{\infty} \frac{n(t)}{t(t+r)} d t
$$

which becomes, after an integration by parts

$$
\log M(r)=r \int_{0}^{\infty} \frac{N(t)}{(t+r)^{2}} d t .
$$

Now let $r=e^{x}, t=e^{y}$, and define $m(x), M(x)$ and $G(x)$ by $m(x)=n\left(e^{x}\right), M(x)=N\left(e^{x}\right)$ and $G(x)=\log M\left(e^{x}\right)$. The formulas become

$$
G(x)=\int_{-\infty}^{\infty} k_{1}(x-y) m(y) d y
$$

and

$$
G(x)=\int_{-\infty}^{\infty} k_{2}(x-y) M(y) d y,
$$

with

$$
k_{1}(t)=\left(1+e^{-t}\right)^{-1}
$$

and

$$
k_{2}(t)=\left(e^{t}+2+e^{-t}\right)^{-1} .
$$

Both $m$ and $M$ are positive and increasing, so certainly in $\mathscr{M}^{\prime}$, and $k_{2}$ clearly satisfies the hypotheses of Theorem 1. Since (10.4) states that $G(x) / m(x) \rightarrow L$, Theorem 1 applied to (10.10) yields that $\log M(r)$ satisfies (10.6) and (10.7).

Theorem 1 may not be applied at once to the situation (10.9), as $k_{1}(t)$ is not integrable. However, the condition $f(0) \neq 0$ implies that $m(y)$ vanishes to the left of $y=K$, for some $K$. This leads to a direct proof of Lemma 2, which was the only place where the integrability of $k$ was needed. Hence, (10.6) and (10.7) follow from (10.9) and (10.5).

Finally, (10.8) is merely a restatement of (1.9) with the kernels in (10.11) and (10.12); it can, of course, be established independently by use of a standard contour integral.

11. We now show that the conclusion of Theorem 1 holds when $g$ is defined by

$$
g(x)=\int_{0}^{x} k(x-y) f(y) d y .
$$


THEOREM 6. Let $f \in \mathscr{M}^{\prime}$, and assume that $k(t)$ is in $L_{1}$ and

$$
k(t)>0 \text {, a.e., } \quad 0 \leqq t<\infty,
$$

and let

$$
\int_{0}^{\infty} e^{s t} k(t) d t
$$

be finite only when $-\sigma<s$, for some $\sigma, 0<\sigma \leqq \infty$. Then from

$$
\int_{0}^{x} k(x-y) f(y) d y=\{L+o(1)\} f(x) \quad(x \rightarrow \infty),
$$

with $L>0$, follows that

$$
f(x)=e^{\lambda x} \psi(x)
$$

where

$$
\text { for every fixed } a, \psi(x+a) / \psi(x) \rightarrow 1, \quad(x \rightarrow \infty) .
$$

In addition, $\lambda(\geqq 0)$ satisfies

$$
\int_{0}^{\infty} e^{-\lambda t} k(t) d t=L
$$

REMARK. It is necessary to assume explicitly that $L>0 ;(11.3)$ is satisfied with $L=0$ when $f(x)=e^{x^{2}}$ and $k$ is any $L_{1}$ function.

The arguments of $\$ 2-7$ apply with minor modifications to this situation; we sketch the details. $\$ 2$ deals only with properties of functions in $\mathscr{M}^{\prime}$, so may be carried over verbatim. However, the notion of exponential peaks must be revised:

Definition. Let $\alpha$ and $a$ be real numbers. A sequence $\left\{r_{m}\right\}_{m=1}^{\infty}$ tending to $\infty$ is a sequence of upper $\alpha$-quasi-exponential peaks for $f$ if

$$
f(t) \leqq f\left(r_{m}\right) e^{\alpha\left(t-r_{m}\right)}(1+o(1))
$$

uniformly as $t \rightarrow \infty$ in the interval

$$
-m a+r_{m} \leqq t \leqq r_{m} .
$$

Dually a sequence $\left\{s_{m}\right\}$ tending to infinity is a sequence of lower $\alpha$-quasi-exponential peaks for $f$ if

$$
f(t) \geqq f\left(s_{m}\right) e^{\alpha\left(t-s_{m}\right)}(1+o(1))
$$

uniformly as $t \rightarrow \infty$ in the interval

$$
-m a+s_{m} \leqq t \leqq s_{m}
$$

The major revision is that (11.6) and (11.8) only hold on one side of the peaks (11.7) and (11.9). This is not surprising, since (11.1) shows that $g(x)$ depends on the behavior of $f(y)$ only for $y \leqq x$. Theorems 2 (with quasi-exponential peaks instead 
of exponential peaks) and 3 and the proof of Theorem 1 are all applicable; the proof of Theorem 3 in this context is easily adapted from the discussion of \$3. A major simplification is that the discussion in $\$ 3$ culminating in Lemma 5 is unnecessary as the form (11.1) shows.

The analogues of the results of $\$ 4$ are valid also, but the order of discussion and the proofs must be revised.

LEMMA 11. If the hypotheses of Theorem 6 are satisfied, then for each $a>0$ there exist $K$ and $x_{0}$ such that

$$
\begin{array}{ll}
f(t) / f(x)<K, & x_{0}<x \leqq t \leqq x+a, \\
g(t) / g(x)<K, & x_{0}<x \leqq t \leqq x+a .
\end{array}
$$

Proof. It suffices to prove the lemma only when $a$ is sufficiently small; we assume throughout the proof that $a<a_{0}$, determined by (1.3). Choose $x_{1}$ with the property that

$$
\begin{aligned}
& f(t) / f(x)>\varepsilon, \\
& g(t) / g(x)>\varepsilon
\end{aligned}
$$

when $x_{1}<x \leqq t \leqq x+a$, and $a<a_{0}$. Then, using Lemma 2 ,

$$
\begin{aligned}
g(x) & =\int_{0}^{x} k(x-y) f(y) d y \\
& >\varepsilon \int_{x_{1}+a}^{x} k(x-y) f(y-a) d y+\int_{0}^{x_{1}} k(x-y) f(y) d y \\
& =\varepsilon \int_{0}^{x} k(x-y) f(y-a) d y+o(g(x)),
\end{aligned}
$$

so

$$
g(x)>\varepsilon \int_{0}^{x-a} k(x+a-y) f(y) d y+o(g(x)) .
$$

On the other hand, from (11.12)

$$
\int_{x-a}^{x+a} k(x+a-y) f(y) d y<e^{-2} f(x+a)\left\{\int_{0}^{2 a} k(t) d t\right\},
$$

so combining (11.14) and (11.15) leads to

$$
\begin{aligned}
g(x+a) & =\int_{0}^{x+a} k(x+a-y) f(y) d y \\
& <\left(\varepsilon^{-1}+o(1)\right) g(x)+\left\{\int_{0}^{2 a} k(t) d t\right\} \varepsilon^{-2} f(x+a) \\
& <\left(\varepsilon^{-1}+o(1)\right) g(x)+\left\{\int_{0}^{2 a} k(t) d t\right\} 2\left(\varepsilon^{2} L\right)^{-1} g(x+a) .
\end{aligned}
$$


Since $\varepsilon$ is independent of $a$, so long as $a<a_{0}$, the lemma follows from choosing $a$ with

$$
\int_{0}^{2 a} k(t) d t<\frac{1}{2} \varepsilon^{2} L .
$$

The proof of the stronger property that given $\eta>0$ there exist $\nu>0$ with

$$
f(t) / f(x)<1+\eta, \quad g(t) / g(x)<1+\eta,
$$

when $x_{0}<x \leqq t \leqq x+\nu$ is easily adapted from the corresponding derivation in $\S 5$.

The lemma of $\$ 6$ appears as

LeMMA 12. Let $a>0$ be fixed and define $N(=N(a))$ and $\nu(=\nu(a))$ by

$$
\begin{aligned}
& N=\limsup _{x \rightarrow \infty} g(x+a) / g(x) \quad(<\infty), \\
& \nu=\liminf _{x \rightarrow \infty} g(x+a) / g(x) \quad(>0) .
\end{aligned}
$$

Consider sequences $\left\{x_{k}\right\}$ and $\left\{y_{k}\right\}$ tending to infinity with

$$
g\left(x_{k}+a\right) / g\left(x_{k}\right) \rightarrow N, \quad g\left(y_{k}+a\right) / g\left(y_{k}\right) \rightarrow \nu .
$$

I. Then, given $A(>0)$ and $\varepsilon(>0)$,

$$
g(s+a) / g(s)>N-\varepsilon
$$

whenever

$$
x_{k}-A \leqq s \leqq x_{k}, \quad k>K_{0}(A, \varepsilon, a) .
$$

II. Similarly,

$$
g(s+a) / g(s)<\nu+\varepsilon
$$

whenever

$$
y_{k}-A \leqq s \leqq y_{k}, \quad k>K_{0}(A, \varepsilon, a) .
$$

The proof of this lemma as well as the construction of the relevant exponential peaks, follows as did their counterparts in $\$ 66$ and 7 . The examples of $\S 8$ apply also to the present situation.

\section{BIBLIOGRAPHY}

1. A. Edrei and W. H. J. Fuchs, The deficiencies of meromorphic functions of order less than one, Duke Math. J. 27 (1960), 233-250.

2. _- "Tauberian theorems for a class of meromorphic functions with negative zeroes and positive poles," pp. 339-358, in Contemporary problems of the theory of analytic functions, "Nauka", Moscow, 1966.

3. W. Feller, On the classical Tauberian theorems, Arch. Math. 14 (1963), 317-322.

4. - An introduction to probability theory and its applications, Vol. II, Wiley, New York, 1966, pp. 418-422.

5. G. H. Hardy and W. W. Rogosinski, Notes on Fourier series. III, Quart. J. Math. Oxford Ser. 16 (1945), 49-58. 
6. J. Karamata, Sur un mode de croissance régulière des fonctions, Mathematica (Cluj) 4 (1930), 38-53.

7. —_ Neuer Beweis und Verallgemeinerung einiger Tauberian-Sätze, Math Z. 33 (1931), 294-299.

8. E. C. Titchmarsh, The theory of functions, Oxford Univ. Press, New York, 1939, p. 271.

9. G. Valiron, Sur les fonctions entieres d'ordre fini et d'ordre null, et en particulier les fonctions a correspondance régulière, Ann. Fac. Sci. Univ. Toulouse (3) 5 (1913), 117-257.

10. D. Widder, The Laplace transform, Princeton Univ. Press, Princeton, N. J., 1941, p. 244.

Purdue University,

LAFAYETTE, INDIANA 